\title{
Análisis de las competencias de pensamiento critico desde el aborde de las cuestiones sociocientificas: un estudio en el ámbito universitario
}

\author{
Solbes, J. ${ }^{1}$, Torres, $\mathbf{N} .^{2}$
}

(1) Dpto. de Didáctica de las Ciencias Experimentales y Sociales. Universitat de València. Jordi.solbes@uv.es (2) Facultad de Educación. Universidad Pedagógica y Tecnológica de Colombia. yanethtorres3@hotmail.com

Resumen: En este trabajo se presenta un análisis acerca de las competencias que promueven el pensamiento crítico a partir del aborde de una cuestión socio-científica, relacionada con la privatización de una empresa de energía eléctrica. En tal sentido, se hace un análisis de estas competencias que son abordadas desde distintas categorías de análisis lo cual permite evidenciar los enfoques que tienen los estudiantes y que influirían en el sentido dado a este tipo de pensamiento. Los resultados señalan la necesidad de implementar estrategias didácticas que favorezcan el desarrollo y fortalecimiento de este tipo de competencias críticas en estudiantes del ámbito universitario.

Palabras clave: Pensamiento crítico, cuestiones socio científicas CSC.

Abstract: This paper presents an analysis of the skills that promote this critical thinking from addressing socio-scientific issue related to the privatization of the energy company. In this regard, an analysis of these competencies that are addressed from different categories of analysis that reveal the approaches that have students and influence the meaning given to this thought. The results indicate the need to implement teaching strategies that support the development and strengthening of such competencies sphere of the University.

Key Words: Critical thinking, socio-scientific issues SSI.

(Fecha de recepción: marzo, 2012, y de aceptación: septiembre, 2012)

DOI: 10.7203/DCES.26.1928 


\section{Introduccion}

La inmersión del estudiante y del docente en escenarios con exigencias sociales necesariamente prioriza la preparación para actuar de manera crítica; por ello desde las últimas décadas diversos estudios promueven una educación participativa, libre, formadora de ciudadanía, que permita a los educandos construir sus conocimientos coherentemente y a los docentes que diseñen e implementen estrategias didácticas que se articulen con la realidad y la cotidianidad.

Una manera de contribuir a lo anterior es promover el desarrollo del pensamiento crítico en todos los procesos educativos, implementando en la enseñanza de las ciencias las cuestiones socio-científicas como estrategia didáctica para motivar este tipo de pensamiento. Desde esta mirada la enseñanza de la ciencia se convierte en una apuesta que posibilite una participación activa de los estudiantes, involucrándose en discusiones públicas sobre situaciones de interés colectivo y social acerca de aspectos legales, políticos y ambientales, lo que, a su vez, da elementos de racionalidad crítica frente a todos los debates sociales y fomenta la conciencia por la resposabilidad social.

Consideramos que la utilización de actividades didácticas como las cuestiones socio-científicas permiten a los estudiantes estructurar su pensamiento crítico, es decir, una manera de pensar propia que les permite distinguir lo verdadero de lo falso, tomar posiciones frente a las situaciones sociales y tener un papel activo en las decisiones culturales y científicas.

En ese sentido se hace un llamado a que los educadores formulen estrategias didácticas que proporcionen a los estudiantes las herramientas necesarias para avanzar en el desarrollo del pensamiento crítico, de manera que se constituyan espacios donde los estudiantes sean protagonistas en la construcción de su propio conocimiento y se les permita combinar competencias para actuar de manera dinámica en los procesos escolares y sociales.

Con el fin de contribuir a lo anterior, se presenta aquí el primer avance de una investigación más amplia relacionada con el desarrollo del pensamiento crítico a partir de la utilización de las cuestiones socio-científicas, donde se plantea el problema de las competencias que tienen los estudiantes de Licenciatura en Ciencias Naturales y Educación Ambiental de una Universidad Colombiana respecto al pensamiento crítico.

En consonancia con lo anterior, en esta investigación, nos planteamos el siguiente problema:

- ¿Qué competencias de pensamiento crítico tienen los estudiantes universitarios en el ámbito de cuestiones sociocientíficas?

Para dar respuesta a anterior cuestionamiento nos planteamos la siguiente hipótesis:

- Los estudiantes tienen un bajo nivel de competencias críticas debido a que es un procedimiento complejo, dificil de aprender, que requiere de muchas competencias "previas". 
En relación con el problema y la hipótesis presentada anteriormente, se hizo necesario examinar el concepto de pensamiento crítico, en una bibliografía que permita identificar los enfoques y pensar propuestas didácticas que promuevan el desarrollo del pensamiento crítico para a partir de allí plantear nuestro propio concepto de pensamiento crítico.

\section{Marco teorico}

El concepto de pensamiento crítico, ha tenido una amplia trayectoria en las últimas décadas, así por ejemplo estudios de: Perry (1970), Kincheloe ( 2000), Ennis (1985) asumen el pensamiento crítico en relación con el uso eficaz de habilidades para la toma de decisiones; Henderson y Landesman (1991) relacionan el pensamiento crítico con el desarrollo de la identidad; Damarin (1993) asume el pensamiento crítico en relación con conocimientos incompatibles o paradójicos; y Cambers et al (2000) señalan que el pensamiento crítico proporciona a los estudiantes las herramientas necesarias para saber el tipo de conocimiento que deben utilizar en determinada situación y se proponen analizar o evaluar la estructura y consistencia de los razonamientos, particularmente opiniones o afirmaciones, que la gente acepta como verdaderas en el contexto de la vida cotidiana.

Frente a las competencias del pensamiento crítico, diversos autores (Aikenhead, 2005; Jiménez-Aleixandre, 2010; Solbes \& Vilches, 1997; Sadler, 2009; Solomon, 1996) plantean la necesidad de comprender la ciencia como actividad humana con múltiples relaciones con la tecnología, la sociedad y el ambiente y asumir la existencia de problemáticas socio-científicas es decir, controversias sociales que tienen su base en nociones científicas. Esto supone estudiar el problema SC de manera integral, en su complejidad, de manera que se involucren dimensiones científicas y técnicas y, además, éticas, culturales, filosóficas, sociales, económicas, ambientales, etc.

Las cuestiones socio-científicas de acuerdo a Zeidler et al. (2002), Cross \& Price (1996), Jiménez-Liso et al. (2010), permiten evaluar el desempeño profesional y apoyan la formación de ciudadanos que participen en forma activa y fundamentada en la sociedad. Estas contribuyen no solo asumir papeles de la comunidad científica sino también asumir roles de la sociedad en general por lo cual los estudiantes proponen distintas explicaciones a diversos cuestionamientos que les permiten prepararse para enfrentar de manera correcta diversas problemáticas.

Las cuestiones socio-científicas acceden a la profundización de conceptos representativos con efectos en aspectos sociales, económicos, culturales y promueve reflexiones sobre la finalidad de la enseñanza de una disciplina con contenidos que permitan desarrollar actitudes acerca del desempeño profesional individual y colectivo en la sociedad.

En este sentido, es importante diseñar actividades que permitan utilizar temas de actualidad para evidenciar aspectos específicos que involucran competencias propias del futuro profesional de la educación con un interés 
particular en una sociedad mejor. Así, Dillon (1994), Aikenhead (2005), Ratcliffe \& Grace (2003) y Solbes et al (2010) manifiestan que el abordaje de cualquier disciplina debe considerar elementos controversiales que permitan desarrollar habilidades de argumentación, toma de decisiones, trabajo cooperativo, además de utilizar una metodología para desarrollar pensamiento crítico e independencia intelectual.

Reis \& Galvão (2004), Sadler (2009), Ramsey (1993), Pedretti (1995), Cross \& Price (1996) y Solomon (1996), afirman de la misma manera que la utilización de cuestiones socio-científicas hace que los estudiantes enfrenten conflictos, formulen opiniones y tomen decisiones.

Por otra parte la fundamentación teórica de este estudio se toma desde una perspectiva de la filosofía crítica y la didáctica de las ciencias. Así desde la filosofía, en esencia, se manifiesta que la principal característica del pensamiento crítico es la duda, la sospecha, el escepticismo, aplicado a todo y, en particular, a los discursos y/o acciones que reproducen y legitiman lo ya establecido. De la misma manera, el abordaje filosófico contribuye a cuestionar la concepción cientificista de la ciencia que se muestra como verdadera y sin problemas y se considera incuestionable.

En este sentido, se apoya en los planteamientos de filósofos de la teoría crítica o escuela de Frankfurt como Habermas (1972) o Marcuse (1994) que, desde una teoría crítica radical, aportan elementos que revelan la función del lenguaje como "ocultador" de la realidad, que permiten exhibir las falsedades que ocultan una realidad y la profunda alienación que gobierna la realidad individual y social de la "civilización industrial", mostrándola como un aparato negador de las verdaderas necesidades humanas, de las posibilidades de liberación a que aspira el ser humano y la sociedad.

Respecto de la didáctica de las ciencias los estudios de Vieira y Nascimento (2007), Jimenez-Aleixandre (2010) Vílchez (2009), Freire (2002), Solbes y Vilches (1997 y 2004), Kolstø (2006), Newton et al. (1999); se toman elementos que permiten cuestionarnos acerca de la necesidad de implicarnos en discusiones públicas acerca de asuntos importantes que se relacionan con la ciencia y la tecnología. Estos autores, coinciden en que al trabajar una cuestión problemática es posible mostrar el papel social de la ciencia tanto en sus aspectos internos como externos, lo cual permitirá el desarrollo del pensamiento crítico.

Consideramos que desde las perspectivas anteriores se promoverá en los estudiantes la comprensión de los fenómenos cotidianos, descubriendo alternativas a su alcance para participar en la toma de decisiones. Así mismo, la discusión de cuestiones socio-científicas en el aula de clase puede ser un paso importante para la formación de ciudadanos que entiendan la naturaleza de la ciencia, sus aplicaciones, sus implicaciones sociales y las políticas del conocimiento científico. Junto con eso es necesario que la escuela forme en los conocimientos científicos mínimos para que los ciudadanos tengan condiciones de juzgar el contexto científico en el que están insertados y tomar decisiones fundamentadas (Sadler et al. 2004). 
Es importante que desde la educación científica se pronuncien esfuerzos didácticos hacia el desarrollo del pensamiento crítico, dado que en la actualidad muchas de las noticias trasmitidas por los medios de comunicación tienen que ver con temas de ciencia, por lo que si no se tiene este tipo de pensamiento las personas se quedan simplemente con opiniones dominantes que engañan y no muestran la verdad de las cosas.

Pensar la enseñanza con la óptica del pensamiento crítico implica a su vez considerar los intereses propios de los estudiantes, lo que requiere que el docente investigue a fondo el contexto social, cultural y político de las comunidades a la cual pertenecen sus estudiantes.

Pero ante todo hay que considerar que establecer relaciones entre los conocimientos científicos y los procesos cotidianos no es una relación simple; requiere de un nivel de comprensión y claridad tanto del léxico científico como del uso cotidiano del lenguaje para poder establecer analogías pertinentes y ejemplos claros. Atendiendo a lo anterior, se eligió una cuestión controversial del contexto relacionada con la privatización de la empresa de Energía de Boyacá que permitió diseñar la prueba diagnóstica con la finalidad de caracterizar las competencias del pensamiento crítico en los estudiantes participantes en el estudio.

\section{Metodologia de la investigacion}

Para la caracterización de las competencias y abordar el problema plantea- do, se diseñó un test (Anexo A) relacionado con la privatización de la empresa de Energía de Boyacá Colombia y se informo a los estudiantes el propósito de este instrumento, se dispuso de una hora y treinta minutos, recomendándoles contestar con la mayor sinceridad posible.

Para efectos del análisis de los datos suministrados por este instrumento se realizaran dos niveles de interpretación, uno que corresponde a mirar cuantitativamente el porcentaje de estudiantes que responden a las preguntas y que dará cuentas de las competencias y el otro realizando descripción textual de las respuestas dadas por los estudiantes. En la tabla 1, se presentan las competencias establecidas para el pensamiento crítico según la investigación que adelantan los autores de este estudio; las dificultades de los estudiantes y las preguntas del pre test (Anexo A) fueron direccionadas a recoger información de cada competencia lo que permitió obtener el porcentaje alcanzado de cada una en los grupos de estudio.

Se utilizaron las siguientes categorías con el fin de sistematizar cada una de las preguntas:

\section{Tabla 2. Criterios de sistematización en el pre test}

Criterio

No responden

Explicación Adecuada

Explicación fuera de contexto

Explicación con rasgos generales
Denomin.

NR

EA

EF

ERG 


\section{Tabla 1. Competencias de pensamiento crítico y dificultades para conseguirlo}

\section{Una persona con pensamiento crítico que aborde CS demandaría:}

\author{
Dificultades que impiden \\ el PC
}

Item

III.1,

III.2. miento de elite, lejano de ellos y descontextualizado. No ser conscientes de los problemas a los que se enfrenta hoy la humanidad, ni del papel de la ciencia y la tecnología en los mismos.

2. Tener disposición para cuestionar las II.2, III.4, das en discursos legitimadores (o en la III.5 ideología dominante) y desconocimiento de los intereses subyacentes.

clusiones no basadas en pruebas, detectar falacias argumentativas, evaluar la credibilidad de las fuentes teniendo en cuenta los intereses subyacentes.

3. Estudiar el problema SC de manera integral, en su complejidad, de manera que se involucren dimensiones científicas, técnicas, éticas, culturales, sociales económicas, ambientales, etc.
3. Estudiar el problema SC de manera I.1, integral, en su complejidad, de manera que se involucren dimensiones científicas, técnicas, éticas, culturales, sociales económicas, ambientales, etc.
4. Valorar y realizar juicios éticos en torno a la CSC atendiendo a la contribución de los mismos a la satisfacción de necesidades humanas, a la solución de los problemas del mundo.
4. Valorar y realizar juicios éticos en III.3, torno a la CSC atendiendo a la contriIII. 6 bución de los mismos a la satisfacción de necesidades humanas, a la solución de los problemas del mundo.

5. Llegar a conclusiones que lleven a tomar decisiones fundamentadas y a promover acciones para el mejoramiento de la calidad de vida y que son capaces de transformar su realidad solucionando diferentes situaciones a nivel personal, familiar y laboral.

\section{Resultados y analisis}

Caracterización de las competencias de pensamiento crítico

En la caracterización de las competencias propias del pensamiento crítico participaron 57 estudiantes, distribuidos en dos grupos, el primero conformado por 31 estudiantes que cursan la asignatura Química General de primer semestre y el segundo corresponde a 26 estudiantes que cursan la asignatura Didáctica General de sexto semestre. 
Ambos grupos pertenecen a la Licenciatura en Ciencias Naturales y Educación Ambiental de la Universidad Pedagógica y Tecnológica de Colombia (UPTC).

Comparando las respuestas de los dos grupos se evidencia que los estudiantes obtienen valores por debajo del $50 \%$ en este tipo de competencias. También se constata que los estudiantes del grupo de didáctica obtienen porcentajes mayores en cada una de estas (Anexo A). Esto se puede explicar considerando que los estudiantes del grupo de didáctica cursan sexto semestre de la licenciatura y han cursado asignaturas relacionadas con aspectos sociales y ambientales como la Educación ambiental. En el grupo de química se observó este orden de dificultad de cada una de las competencias: $5 \leq 3 \leq 2 \leq 4 \leq 1$, mientras que en el grupo de Didáctica se evidencia un orden de dificultad muy similar: $5 \leq 2 \leq 3 \leq 4 \leq 1$.

Los resultados también muestran que la competencia N.5 es la que menor valor obtiene; considerando que los estudiantes de los dos grupos no responden en su mayoría las preguntas. En el caso de los estudiantes del grupo de química el $71 \%$ NR o manifiestan respuestas fuera de contexto mientras que los estudiantes de Didáctica el NR alcanza un $54 \%$. En esta competencia es evidente que los estudiantes tienden a dar opiniones alejadas de su acción personal, es decir, no destacan la manera como cada individuo pueda hacerse participe de la CS.

Frente a la competencia N. 1, que tiene como propósito comprender la ciencia como actividad humana con múltiples relaciones con la tecnología, la sociedad y el ambiente y asumir la existencia de problemáticas socio científicas, es decir, controversias sociales que tienen su base en nociones científicas, los estudiantes reconocen sin dificultad que el problema es la privatización de la empresa, sin embargo, muchos no relacionan la cuestión de la privatización con el estudio de la ciencia, lo que demuestra una brecha en comprender la ciencia como actividad social en la que también se discuten escenarios de poder y de economía condicionados a épocas y contextos actuales.

En la competencia N.2, que tiene por objeto caracterizar que tan informados están los estudiantes sobre el tema, se evidencia que frente a la afirmación presentada en el Pretest: "Se afirma que las centrales hidroeléctricas no son contaminantes, ayudan a frenar la emisión de $\mathrm{CO}_{2}$ y no producen impactos ambientales" no hay cuestionamiento de su veracidad (esto es más evidente en estudiantes del grupo de Química) y aceptan la afirmación sin evaluar su credibilidad.

La competencia N.3, corresponde al estudio del problema SC de manera integral, en su complejidad, de manera que se involucren dimensiones científicas y técnicas y, además, éticas, culturales, filosóficas, sociales, económicas, ambientales, etc.

Se detecta que los estudiantes solo transcriben lo que ven en la imagen, sin interpretar el proceso efectuado para la generación de la energía eléctrica y, además, manifiestan no conocer otros procedimientos que permitan hacer girar la turbina y generar la energía eléctrica. En la tercera pregunta de 
esta competencia relacionada con la producción de energía en Boyacá los estudiantes manifiestan que la produce la empresa EBSA pero desconocen todo el proceso que ocurre para la generación de la energía eléctrica; no explican la procedencia del combustible y el proceso como tal de la producción de la energía eléctrica.

Esta competencia es la segunda con valores más bajos en el grupo de química y la tercera en el grupo de didáctica, lo cual hace evidente la dificultad que tienen los estudiantes al estudiar el problema de manera integral.

En la competencia N. 4; que tiene como finalidad caracterizar si los estudiantes son capaces de realizar juicios éticos en torno a la contribución de la implementación de la energía eléctrica en el país, muchos optaron por no responder esta pregunta y otros argumentaban solo destacando la importancia económica de la energía eléctrica en el país: La valoración de la energía eléctrica no es muy adecuada y eficiente para la alta producción en el país.

Sin embargo, también se evidencia que responden con facilidad nuevamente la pregunta relacionada con la privatización, pero los estudiantes no suelen realizar juicios éticos y sólidos respecto a CS.

Lo anterior ponen de manifestó el bajo nivel alcanzado por los estudiantes en cada una de las competencias críticas, considerando que se requieren escenarios didácticos que las promuevan, que permitan la conexión entre la ciencia y la sociedad, el cuestionamiento de la información, la comprobación de la validez de los argumentos y la consolidación de una formación integral enmarcada en la participación ciudadana, la formación en valores y la toma de decisiones fundamentadas.

A continuación se señalan en la tabla 3 los porcentajes de explicaciones adecuadas (EA) alcanzados por cada competencia en los grupos de estudiantes participantes:

\section{Tabla 3. \% Alcanzados en cada competencia}

\begin{tabular}{lcc}
$\begin{array}{l}\text { Competencias } \\
\text { de Pensamiento } \\
\text { Critico }\end{array}$ & $\begin{array}{c}\text { Est. } \\
\text { Química } \\
(\%)\end{array}$ & $\begin{array}{c}\text { Est. } \\
\text { Didáctica } \\
(\%)\end{array}$ \\
\hline & 37 & 44 \\
2 & 13 & 21 \\
3 & 7 & 25 \\
4 & 33 & 38 \\
5 & 0 & 11 \\
\hline
\end{tabular}

Para el análisis por competencias individuales se consideraron el número de estudiantes que realizan una explicación adecuada (EA), una explicación con rasgos generales (ERG), el número de estudiantes que no responden (NR) y los que realizan una explicación fuera de contexto de cada grupo de estudio $(\mathrm{EF})$, criterios también presentados en la tabla N. 2.

Primera competencia: (Tabla 4)

En la primera pregunta un 5\% de la totalidad del grupo de estudiantes no responden (NR) y un $13.8 \%$ manifiestan que no es claro el problema, por lo que argumentan en sus respuestas opiniones como: Son simplemente problemas de orden económico, puesto que dicen que la nación no tiene presupuesto para 


\section{Tabla 4: \% de respuestas en la competencia 1}

\begin{tabular}{|l|c|c|c|c|c|c|c|c|}
\hline CURSO & \multicolumn{3}{|c|}{$\begin{array}{c}\text { ¿Identificas problemas en lo } \\
\text { anteriormente descrito? } \\
\text { En caso afirmativo } \\
\text { ¿Cuáles y Por qué? (\%) }\end{array}$} & \multicolumn{2}{c|}{$\begin{array}{c}\text { Consideras que la situación } \\
\text { descrita anteriormente } \\
\text { puede ser objeto de reflexión } \\
\text { de las ciencias (\%). }\end{array}$} \\
\cline { 2 - 9 } & EA & ERG & NR & EF & EA & ERG & NR & EF \\
\hline QUIMICA & $(17)$ & $(7)$ & $(2)$ & $(5)$ & $(6)$ & $(7)$ & $(18)$ & $(0)$ \\
& 54,8 & 22,5 & 6,4 & 16,1 & 19,3 & 22,5 & 58,0 & 0,0 \\
\hline DIDACTICA & $(18)$ & $(4)$ & $(1)$ & $(3)$ & $(5)$ & $(6)$ & $(10)$ & $(5)$ \\
& 69,2 & 15,3 & 3,8 & 11,5 & 19,2 & 23,0 & 38,4 & 19,2 \\
\hline
\end{tabular}

mantener el servicio y por esto busca la ayuda de inversionistas privados.

La anterior afirmación evidencia que solo un mínimo porcentaje relaciona la privatización con conceptos de economía, sin embargo en la segunda pregunta que buscaba evidenciar si los estudiantes articulaban esa situación como objeto de reflexión de la ciencias se observo que la mayoría de los estudiantes 58\% (Química) y 38,4 (Didáctica) no responden la pregunta, lo que se muestra la falta articulación de cuestiones sociales con los temas de ciencia, viendo esta última como un conjunto de ecuaciones y fórmulas y no su influencia en la sociedad y el ambiente.

No obstante de los estudiantes que responden afirmativamente a esta pregunta se manifestaron las siguientes respuestas: "Si por que se da la contribución de la ciencia para buscar nuevos materiales que permitan la producción de energía". "Si creo que a pesar de que esta empresa sea patrimonio, primero se deberían crear empresas generado- ras de energía eléctrica más naturales aprovechando más la energía eólica o solar". En estas opiniones se evidencia una noción más social de la ciencia como actividad humana y forma de contribuir a mejorar la calidad de vida y el ambiente.

Llama la atención la respuesta de un estudiante de química quien manifiesta: Si porque falta aplicabilidad de estas en $100 \%$, lo que hace pensar que para este estudiante; la ciencia no tiene aplicabilidad en la cotidianidad y considera la ciencia como algo abstracto, afirmación que nos permitiría discutir el sentido humano de las aplicaciones de la ciencia.

Segunda competencia: (Tabla 5)

En la primera pregunta para esta competencia se pretendía que los estudiantes detectaran la falacia que proponía lo siguiente: Se afirma que las centrales hidroeléctricas no son contaminantes, ayudan a frenar la emisión de $\mathrm{CO}_{2}$ y no producen impactos ambientales. 


\section{Tabla 5: \% de respuestas en la competencia 2.}

\begin{tabular}{|c|c|c|c|c|c|c|c|c|c|c|c|c|}
\hline \multirow[t]{2}{*}{ CURSO } & \multicolumn{4}{|c|}{$\begin{array}{c}\text { ¿Está de acuerdo con } \\
\text { la afirmación anterior? } \\
\text { ¿Cuál ha sido el impacto } \\
\text { de las centrales hidroeléc- } \\
\text { tricas en el ambiente y la } \\
\text { sociedad? 2. ¿Y el de las } \\
\text { térmicas? (\%) }\end{array}$} & \multicolumn{4}{|c|}{$\begin{array}{c}\text { Estás de acuerdo con } \\
\text { la forma de producción } \\
\text { de la energía eléctrica } \\
\text { en el departamento de } \\
\text { Boyacá. \% }\end{array}$} & \multicolumn{4}{|c|}{$\begin{array}{c}\text { ¿Qué controversias o } \\
\text { desacuerdos científicos } \\
\text { relacionados con la } \\
\text { producción y el uso de } \\
\text { la energía eléctrica se } \\
\text { han dado?\% }\end{array}$} \\
\hline & EA & ERG & NR & $\mathrm{EF}$ & EA & ERG & NR & $\mathrm{EF}$ & EA & ERG & NR & $\mathrm{EF}$ \\
\hline \multirow[t]{2}{*}{ QUIMICA } & (3) & (13) & (6) & (9) & (4) & (6) & (13) & (8) & (6) & (2) & (19) & (4) \\
\hline & 9,6 & 42 & 19,3 & 29 & 12,9 & 19,3 & 41,9 & 25,8 & 19,3 & 6,4 & 61,2 & 12,9 \\
\hline \multirow[t]{2}{*}{ DIDACTICA } & (9) & (5) & (4) & (8) & (4) & (7) & (6) & (9) & (4) & (7) & (13) & (2) \\
\hline & 34,6 & 19,2 & 15,3 & 30,7 & 15,3 & 27 & 23 & 34,6 & 15,3 & 27 & 50 & 7,6 \\
\hline
\end{tabular}

Los estudiantes en un $29,8 \%$ no detectan la falacia y argumentaron apoyando la afirmación con opiniones como: "Si estoy de acuerdo con lo anterior, no conozco impactos ambientales de gran magnitud referentes a las hidroeléctricas". "Pues creo que las hidroeléctricas no contaminan casi nada pues no emiten gases". "Estoy de acuerdo que la hidroeléctrica mitiga la contaminación ambiental"; estas opiniones permiten inferir que la falta de información sobre el tema y el no cuestionamiento frente a la validez de los argumentos favorecen la formulación de opiniones acríticas en los estudiante, por lo que es indispensable posibilitar escenarios didácticos de debate, que posibiliten el cuestionamiento de la información antes de su aceptación.

Se evidencia que los estudiantes del grupo de Didáctica argumentan mejor la respuesta que los estudiantes del grupo de química, sin embargo se muestra que no hay claridad de los efectos de las centrales hidroeléctricas y de las centrales térmicas:

Estudiante de Didáctica (ED). No estoy de acuerdo ni en desacuerdo porque todos los procesos tienen impactos buenos y malos, pero lo que sí puedo agregar es que estas empresas si producen impactos ambientales que conllevan a la destrucción de nuestra madre tierra.

Estudiante de Química (EQ). No ya que toda la industria de este tipo producen unos gases.

A pesar de denotar cierto desacuerdo con la información en las anteriores opiniones no se explican efectos de las hidroeléctricas en la población, en los ecosistemas, en los ríos, ni mencionan específicamente los gases que se producen. $\left(\mathrm{HNO}_{3} ; \mathrm{H}_{2} \mathrm{SO}_{4}\right.$ macropartículas (smog); y la influencia en enfermedades cardiorespiratorias. 
En relación a: las centrales hidroeléctricas ayudan a frenar el $\mathbf{C O}_{2}$, el $21 \%$ de los estudiantes reconocen su efecto y ofrecen argumentos como: Las centrales hidroeléctricas pueden ayudar a frenar la emisión del $\mathrm{CO}_{2}$, pero debido a la utilización de grandes cantidades de agua y la retención y posterior evaporación detienen el trayecto de los ríos. Las centrales térmicas generan erosión por la necesidad de explotar el carbón. A pesar de que en esta opinión se manifiesta el acuerdo con la afirmación, no se dan las razones que justiquen la opinión, lo que permiten evidenciar la dificultad que tienen los estudiantes para establecer relaciones entre conceptos de las ciencias y extrapolarlos a contextos escolares y cotidianos.

Un $49 \%$ de la totalidad del grupo dan explicaciones con rasgos generales: No estoy de acuerdo que las centrales hidroeléctricas producen un daño al ambiente porque por el proceso que se llevan producen daño a la atmosfera y a la capa de ozono, esta afirmación señala que los estudiantes acogen conceptos muy usuales porque involucra una temática ambiental, en muchos de los casos son los mismos que emiten los medios de comunicación, y se observa que en esta opinión el estudiante, no menciona los gases producidos por las hidroeléctricas ni especifica las razones del porque afecta a la atmosfera y a la capa de ozono.

Frente a la pregunta acerca de si está de acuerdo con la forma como se produce energía en Boyacá, los estudiantes en un $41,9 \%$ de grupo de Química y un $23 \%$ del grupo de didáctica no respon- den a la pregunta, otros responden NO pero justifican en otro sentido: "No, $y a$ que está contaminando y no han crecido (sic) un verdadero diseño en su estructura". Afirmaciones como esta siguen evidenciando el desvinculamiento que puede haber entre los conceptos de química con situaciones contextuales; los estudiantes manifiestan un No como respuesta, pero no revelan explicaciones lógicas e interesantes argumentadas desde los conceptos de la ciencia, lo que justifica una vez más la importancia de establecer conexiones entre ciencia, educación y sociedad.

Otros al contrario argumentan en sentido diferente, manifestando que son recursos de la naturaleza y que es un beneficio económico para todos "Si pues se aprovechan los recursos de la naturaleza como son el agua y las minas". "Si es un proceso rentable, es propiedad del departamento es económico para todos" Esta afirmación permite relacionar el beneficio económico que trae la explotación de los recursos, se destaca en esta afirmación la vinculación que hacen de los recursos naturales con lo económico, sin embargo habría que cuestionar la manera como los estudiantes ven la ciencia y la tecnología, como un proceso netamente económico, perfecto, sin impactos en el ambiente.

Lo anterior señala que los estudiantes no tienen claridad respecto a la forma de producción de energía en Boyacá, dado que no justifican sus respuestas explicando la obtención del combustible de municipios aledaños, los impactos que trae, la ubicación de la hidroeléctrica y los procesos que se efectúan. 


\section{Tabla 6: \% de respuestas en la competencia 3.}

\begin{tabular}{|c|c|c|c|c|c|c|c|c|c|c|c|c|}
\hline \multirow[t]{2}{*}{ CURSO } & \multicolumn{4}{|c|}{$\begin{array}{l}\text { Observa el esquema } \\
\text { que aparece a la } \\
\text { derecha y describe } \\
\text { el procedimiento } \\
\text { que se requiere para } \\
\text { la generación de la } \\
\text { energía eléctrica. (\%) }\end{array}$} & \multicolumn{4}{|c|}{$\begin{array}{c}\text { ¿Qué otros } \\
\text { procedimientos } \\
\text { conoces que permitan } \\
\text { hacer girar la turbina } \\
\text { para producir energía } \\
\text { eléctrica? (\%) }\end{array}$} & \multicolumn{4}{|c|}{$\begin{array}{c}\text { ¿Cómo se produce } \\
\text { la energía eléctrica } \\
\text { en Boyacá y quien la } \\
\text { produce? }\end{array}$} \\
\hline & EA & ERG & NR & $\mathrm{EF}$ & EA & ERG & NR & $\mathrm{EF}$ & EA & ERG & NR & $\mathrm{EF}$ \\
\hline \multirow[t]{2}{*}{ QUIMICA } & (1) & (11) & (8) & (11) & (2) & (5) & (21) & (3) & (4) & (7) & (15) & (5) \\
\hline & 3,2 & 35,4 & 25,8 & 35,4 & 6,4 & 16,1 & 67,7 & 9,6 & 12,9 & 22,5 & 48,3 & 16,1 \\
\hline \multirow[t]{2}{*}{ DIDACTICA } & (6) & (17) & & (3) & (4) & (3) & (16) & (3) & (10) & (8) & (5) & (3) \\
\hline & 23 & 54,8 & & 11,3 & 15,3 & 11,3 & 61,5 & 11,3 & 38,4 & 30,7 & 19,2 & 7,6 \\
\hline
\end{tabular}

Los estudiantes que responden EA (Explicación Adecuada) manifiestan que se deberían implementar formas de producción de energía más limpias: "No estoy de acuerdo, dado que hay generación de $\mathrm{CO}_{2}$, la explotación de carbono y coke para calentar las cámaras de combustión generando desechos y la transformación del hábitat de las zonas que han sido explotadas". "No, ya que el medio ambiente se ve altamente afectado con la emisión de gases y residuos al medio ambiente". Estas afirmaciones muestran elementos comunes del impacto de las centrales hidroeléctricas en el ambiente.

Frente a la última pregunta relacionada con las controversias sobre el uso y la producción de energía eléctrica llama la atención que los estudiantes $61,2 \%$ (Química) y $50 \%$ (Didáctica), no tienen conocimiento acerca de las discusiones relacionadas con asuntos científicos y un $17,5 \%$ de la totalidad del grupo manifestó como controversia común: La Emi- sión de gases y la utilización de recursos no renovables, esto permite inferir que los estudiantes han concebido una idea lineal de la ciencia en la que el conocimiento se ha desarrollado solo a base de éxitos, y no se ha cuestionado los aspectos internos y externos en la construcción de la ciencia.

Tercera competencia: (Tabla 6) En la tabla anterior los datos muestran que los estudiantes no relacionan conceptos químicos con el esquema presentado, por lo que describen el dibujo con las palabras que la figura muestra, no explican el procedimiento que se efectúa en la generación de la energía eléctrica y hacen una transcripción textual de la imagen, sin interpretar a profundidad los procesos que se desarrollan.

Algunos estudiantes manifestaron que lo que hace mover la turbina es la entrada del agua fría: Gracias a una cámara de combustión que permite la entrada de combustible ya que un condensador que permite la entrada del 
agua caliente, puede entrar agua fría la cual hace girar la turbina de vapor y generar electricidad. No explican la producción de reacciones de combustión de carbón, petróleo o gas que calientan un líquido a mucha a presión, que hace girar bobinas en el interior de un campo magnético (generador), produciendo corriente alterna según la ley de Faraday.

Otros estudiantes describen la imagen sin explicar el procedimiento: "Combustible - cámara de combustible- generador de vapor-turbina de vapor-generador....." "Se necesita de combustible para generar energía, al realizarse este proceso se liberan residuos que es expulsado. Este tipo de respuestas demuestra la dificultad para establecen relaciones entre conceptos de ciencia con procesos cotidianos, desde esa mirada se seguiría observando la ciencia como especialidades (productos individuales) sin relaciones con la sociedad y el ambiente, por lo que se deben promover espacios académicos que permitan estableciendo el mayor número de relaciones con otros campos de conocimiento para evitar, de esta manera visiones reducidas del conocimiento.

En algunas respuestas los estudiante transfieren la producción de energía a consecuencias en el ambiente: Primero hay que extraer la materia prima de la naturaleza que posteriormente va a servir como combustible el cual va a entrar en contacto con los instrumentos tecnológicos necesarios para la producción de energía eléctrica, pero además de generar gases contaminantes al medio ambiente produce un agotamiento irreversible de los ecosistemas. En lo anterior se hace evidente que el estudiante relaciona el proceso de la producción de la energía con el deterioro de los ecosistemas, lo que favorece de cierta manera una comprensión que permita involucrarse en la solución de problemáticas ambientales.

En la segunda pregunta relacionada con otros procedimientos que permitan hacer girar la turbina para producir energía eléctrica, llama la atención que los estudiantes en un 67,7 \% (Química) y el $61,5 \%$ (Didáctica) No responden. En su mayoría solo mencionan la energía del viento y del agua pero no referencian otras como la nuclear, la mareomotriz, las fotoceldas etc. "Emisión de calor por quema de carbón (muy costosa y contaminate) por la fuerza del viento haciendo girar una turbina por fuerza del mar con ayuda de las olas"

En la tercera pregunta: ¿Cómo se produce la energía eléctrica en Boyacá y quien la produce? Los estudiantes en su mayoría manifiestan que la produce Termopaipa con la empresa EBSA, esta respuesta manifiesta un desconocimiento de todo el proceso que ocurre para la generación de la energía eléctrica en el departamento de Boyacá (Colombia), dado que no explican la procedencia del combustible y el proceso como tal de la producción de la energía eléctrica.

Cuarta competencia: (Tabla 7)

Frente a la pregunta acerca de si está de acuerdo con la privatización de la empresa solo el 15,7\% de la totalidad del grupo, No responde (NR) o responde en otra dirección (EF). El 57,8 \% de los estudiantes manifiestan no estar de acuerdo con la privatización y justifican coherentemente su afirmación: "No, aunque se hable de la disminución de los costos, todos sabemos que la privati- 


\section{Tabla 7: \% de respuestas en la competencia 4.}

\begin{tabular}{|c|c|c|c|c|c|c|c|c|}
\hline \multirow[t]{2}{*}{ CURSO } & \multicolumn{4}{|c|}{$\begin{array}{c}\text { Estás de acuerdo con la } \\
\text { privatización de la EBSA. } \\
\text { ¿Por qué? (\%) }\end{array}$} & \multicolumn{4}{|c|}{$\begin{array}{l}\text { Suponga que usted es invitado } \\
\text { a un debate donde se discute } \\
\text { la implementación de energía } \\
\text { eléctrica en el país, y se } \\
\text { requiere saber su valoración } \\
\text { profesional respecto a la } \\
\text { temática, ¿cuál sería tu } \\
\text { declaración? }(\%)\end{array}$} \\
\hline & EA & ERG & NR & $\mathrm{EF}$ & EA & ERG & NR & $\mathrm{EF}$ \\
\hline QUIMICA & $\begin{array}{c}(18) \\
58\end{array}$ & $\begin{array}{c}(8) \\
25,8\end{array}$ & $\begin{array}{l}(2) \\
6,4\end{array}$ & $\begin{array}{l}\text { (3) } \\
9,6\end{array}$ & $\begin{array}{l}(3) \\
9,6\end{array}$ & $\begin{array}{c}(5) \\
16,1\end{array}$ & $\begin{array}{l}(17) \\
54,8\end{array}$ & $\begin{array}{c}(6) \\
19,3\end{array}$ \\
\hline DIDACTICA & $\begin{array}{l}(15) \\
56,7\end{array}$ & $\begin{array}{l}\text { (7) } \\
27\end{array}$ & $\begin{array}{c}(3) \\
11,5\end{array}$ & $\begin{array}{l}\text { (1) } \\
3,8\end{array}$ & $\begin{array}{c}(5) \\
19,2\end{array}$ & $\begin{array}{c}(8) \\
30,7\end{array}$ & $\begin{array}{c}(5) \\
19,2\end{array}$ & $\begin{array}{c}(8) \\
30,7\end{array}$ \\
\hline
\end{tabular}

zación es el antónimo de esto, además en manos de otros solo les importa el beneficio económico". "No la EBSA se ha demostrado que puede generar ganancias a un bajo costo, al igual la capacidad de contaminación es menor si solo se proyectara vender servicios a Colombia”. Estas afirmaciones manifiestan una interpretación de la privatización como una transferencia de propiedad o control del sector público al privado, los estudiantes manifiestan una connotación negativa de la privatización y lo vinculan con aumento de precios, política que favorecen a pocos, se evidencia en estas afirmaciones cierta refutación a la consideración de que las empresas públicas son poco eficientes, poco competitivas internacionalmente, con escasa capacidad de innovación tecnológica $\mathrm{y}$ falta de dinamismo empresarial.
En la segunda pregunta de esta competencia se observa que los estudiantes presentan dificultades para sentar posturas frente a la temática y prefieren no responder: 54,8 (Química) y 19, 2 (Didáctica), este último grupo argumenta pero en otra dirección: "La valoración de la energía eléctrica no es muy adecuada y eficiente para la alta producción en el país". "La incrementación del servicio en el país, los terrenos que se utilizan para la formación de esta" No hay una postura crítica desde el licenciado en Ciencias Naturales, que evidencien posicionamiento complejos que involucren aspectos de ciencia, tecnología, economía, cultura y política, basados en criterios pedagógicos, históricos, epistemológicos, sociales y filosóficos sino por el contrario, se muestra una postura reducida con comentarios muy comunes en muchos de los casos escuchados por 
los medios de comunicación, esto destaca la necesidad de promover alternativas didácticas que permitan articular diversidad de elementos para formar al docente de Ciencias en la integralidad.

Solo un $14,4 \%$ de la totalidad del grupo manifiesta opiniones solidas como las siguientes: Que es adecuado aprovechar la energía del sol y el aire para la producción de electricidad, que se debe ampliar la cobertura y que es necesario cambiar las costumbres de consumo excesivo de servicio y dependencia del mismo. En esa opinión los estudiantes establecieron relaciones entre fuentes alternativas más ecológicas de energía y el servicio de este a la humanidad. Es interesante observar como hace una recomendación acerca del consumo excesivo y la dependencia del mismo y resalta la importancia de que la energía eléctrica tenga cobertura dado que es un bien público.

Quinta competencia: (Tabla 8)

\begin{tabular}{|c|c|c|c|c|}
\hline \multicolumn{5}{|c|}{$\begin{array}{c}\text { Tabla 8: } \% \text { de respuestas en la } \\
\text { competencia } V .\end{array}$} \\
\hline \multirow[t]{2}{*}{ CURSO } & \multicolumn{4}{|c|}{$\begin{array}{c}\text { Basándote en la } \\
\text { declaración anterior } \\
\text { que decisiones sería } \\
\text { necesario asumir a } \\
\text { nivel personal, laboral, } \\
\text { y familiar respecto a la } \\
\text { situación anterior. } \%\end{array}$} \\
\hline & EA & ERG & NR & $\mathrm{EF}$ \\
\hline QUIMICA & $(0)$ & $\begin{array}{l}(9) \\
29\end{array}$ & $\begin{array}{c}(17) \\
55\end{array}$ & $\begin{array}{l}(5) \\
16\end{array}$ \\
\hline DIDACTICA & $\begin{array}{c}(3) \\
11,5\end{array}$ & $\begin{array}{c}(9) \\
34,6\end{array}$ & $\begin{array}{c}(9) \\
34,6\end{array}$ & $\begin{array}{c}(5) \\
19,2\end{array}$ \\
\hline
\end{tabular}

En esta competencia es en la que más se manifiestan las opciones NR y EF. Los estudiantes manifiestan opiniones muy generales sin evidenciar, acciones específicas a nivel personal, laboral y familiar: "Concientizar a nuestras familias de el gran problema que estamos pasando y como estamos afectando mucho al planeta"

Sin embargo un $11.5 \%$ del grupo de didáctica proponen acciones específicas se encuentran las siguientes afirmaciones: "Participación política, directa y comprometida en la vigilancia y elección de los responsables de administrar los recursos naturales de nuestro país" "Primero que todo debe ser desde los personal donde se pueda ver una ética responsable que justifique las acciones que se pretenden llevar a cabo desde el aspecto laboral"

En las anteriores afirmaciones mencionan dos aspectos no considerados antes en las respuestas de los estudiantes, lo político y lo ético; sin embargo quedaría abierta la posibilidad a cuestionar la manera de interpretar lo ético y político en estas respuestas y la importancia de apropiarse de la situación y hacerse participe en el problema y su solución, no solo dar opiniones alejadas, generalizadas que no involucran la acción personal.

\section{Conclusiones y perspectivas}

Esta primera caracterización muestra que los estudiantes pueden reconocer con mayor facilidad el problema de la privatización pero no lo vinculan como objeto de reflexión de la ciencia, lo 
que demuestra una fisura en comprender la ciencia como actividad social.

El instrumento utilizado permitió evidenciar que la competencia de mayor dificultad para los estudiantes es la relacionada con la formulación de conclusiones que lleven a tomar decisiones fundamentadas, a promover acciones para el mejoramiento de la calidad de vida y a ser capaces de transformar su realidad solucionando diferentes situaciones a nivel personal, familiar y laboral, dado que se tienden a dar opiniones alejadas de su acción personal, es decir, no destacan la manera como cada individuo pueda hacerse participe de la CS.

Creemos que con la implementación de las cuestiones socio científicas en la enseñanza de ciencia se puede desempeñar un papel relevante que permite fomentar escenarios alternativos, aportando la defensa de los valores humanos, la responsabilidad social y el pensamiento crítico.

Se evidencia que la mayoría reconoce el problema de la privatización y las implicaciones de esta, pero no lo articulan con aspectos de la ciencia, lo cual permite determinar que hace falta implementar estrategias didácticas que orienten al estudiante en la manera de cuestionar la información dada, para que no crean y se queden con la opinión dominante del momento.

Algunos estudiantes solo ven la dimensión económica en la situación presentada, dado que manifiestan Estoy de acuerdo con la producción de energía por que se produce con recursos del medio y de las minas, pero no consideran los efectos e impactos que conllevan este tipo de procedimiento que permitiera utilizar medidas más sostenibles.

Se demuestra cierta marginalización de aspectos sociales, políticos, éticos, ambientales y científicos en el abordaje de la CS. Los estudiantes tienden a ofrecer argumentos muy comunes que podrían estar influenciados por medios de comunicación. Por eso hace falta profundizar en un discurso crítico que permita asumir posturas frente a este tipo de controversias con un nivel de profundidad propia de los licenciados en Ciencias Naturales, que serán los futuros formadores de las generaciones venideras.

Pese a muchos de los estudios sugieren un cambio en la educación científica en todo el mundo, los resultados evidencian que la enseñanza de las ciencias sigue teniendo un carácter instrumental dado que los estudiantes no están familiarizados con la forma en que la ciencia afecta a la vida de las personas ni con la toma de decisiones que requieren de un conocimiento científico.

El diagnóstico realizado en este trabajo nos permite conocer las dificultades que han tenido los estudiantes y, en consecuencia, nos permitirá proponer estrategias que formen a científicos y ciudadanos con pensamiento crítico que usen la ciencia para tomar decisiones fundamentadas.

\section{Bibliografía}

AIKENHEAD, G. (2005). Educación Ciencia-Tecnología-Sociedad (CTS): una buena idea o como quiera que se le llame. Educación Química, 16(2), 114-124. 
CAMBERS, A., CARTER-WELLS, K., BAGWELL, J., PADGET, J., THOMSON, C. (2000). Creative and active strategies to promote critical thinking. En Yea book of the Claremont Reading Conference (pp. 58-69). Claremont, CA: The Claremont Graduate School.

CROSS, R. \& PRICE, R. (1996). Science Teachers' social conscience and the role of controversial issues in the teaching of science. Journal of research in science teaching. 33,(3), 319-333. http:// dx.doi.org/10.1002/(SICI)10982736 (199603)33:3<319::AID TEA5>3.0.CO;2-W

DAMARIN, S. (1993). Educación y conocimientos situados: ¿el viajar o el turismo?. Tecnología educativa 33 (3), 27-32.

DILLON, J. (1994). Using discussion in classrooms. Buckingham: Open University Press.

ENNIS, R. (1985). A logical basis for measuring critical thinking skills. Educational Leadership. 43(2), 4446.

FREIRE, P. (2002). Pedagogia da Autonomia: saberes necessários à prática educativa. 22.ed. São Paulo: Paz e Terra.

HABERMAS, J. (1972). Teoría analítica de la ciencia y dialéctica. In: Adorno, T.; Popper, K.;Dahrendorf, R.; Habermas, J.; Albert, H.; Pilot, H. La disputa del positivismo en la sociología Alemana. Tradução Jacobo Muñoz. Barcelona: Grijalbo.
HENDERSON, R., LANDESMAN, E. (1991). Visualizar los conceptos de precalculus: la representación interactiva vía la tecnología de videodisco. Computadoras y educación 17,195 - 202.

JIMÉNEZ-ALEIXANDRE, M.P. (2010). 10 ideas clave. Competencias en argumentación y uso de pruebas. Barcelona: Graó.

JIMÉNEZ-LISO, M.R.; HERNÁNDEZVILLALOBOS, L. \& LAPETINA, J. (2010). Dificultades y propuestas para utilizar las noticias científicas de la prensa en el aula de ciencias. Eureka Ens. Div. Cienc., 7(1), 107126.

KINCHELOE, J.( 2000). Hacer la idea crítica. Perspectivas en la idea crítica: composiciones por profesores en teoría y práctica, Peter Lang, Nueva York, $23-37$.

KOLST $\varnothing$, S. D. (2006). Patterns in students' argumentation confronted with risk-focused socioscientific issue. International Journal of Science Education, 28 (14), 1689-1716. http://dx.doi. org/10.1080/09500690600560878

MARCUSE, H. (1994) El hombre unidimensional, Barcelona, Ariel.

NEWT ON, P.; DR I VER, R .; OSBORNE, J. (1999). The place of argumentation in the pedagogy of school science. International Journal of Science Education, 21, (5) 553-576. http://dx.doi. org/10.1080/095006999290570

PEDRETTI, E. (2003). Teaching science, technology, society and 
Environment (STSE) education: Preservice Teachers' philosophical and pedagogical landscapes, en Zeidler, D. (eds). The role of moral reasoning on socioscientific issues and discourse in science education, (pp. 219-239). The Netherlands: Kluwer Academic Publishers. http:// dx.doi.org/10.1007/1-4020-4996-X_12 PERRY, W. (1970). Los formularios del W.G. del desarrollo intelectual en los años de la universidad, un plan. Holt, Rinehart, y Winston, Nueva York, 37. RAMSEY, J. (1993). The science education reform movement: Implications for social responsibility. Science Education, 77(2), 235258. http://dx.doi.org/10.1002/ sce.3730770210

RATCLIFFE, M. \& GRACE, M. (2003). Science education for citizenship: teaching socio-scientific issues. Philadelphia: Open University Press. REIS,P.\&GALVÃO,C.(2004).Theimpact of socio-scientific controversies in Portuguese natural science teachers' conceptions and practices. Research in Science Education, 34 (2), 153171. http://dx.doi.org/10.1023/ B:RISE.0000033760.04656.a1

SADLER, T. D. (2009). Socioscientific issues in science education: labels, reasoning, and transfer. Cultural Studies in Science Education, 4,(3) 697-703. http://dx.doi.org/10.1007/ s11422-008-9133-x

SADLER, T.; CHAMBERS, F.; ZEIDLER, D. (2004) Students conceptualizations of thenature of science in response to a socioscientific
issue.Internationaljournalof Science Education, 26, 387-410. http://dx.doi. org/10.1080/0950069032000119456 SOLBES, J., RUIZ, J.J. Y FURIÓ, C. (2010). Debates y argumentación en las clases de física y química. Alambique, 63, 65-76.

SOLBES, J. \& VILCHES, A. (1997). STS interactions and the teaching of physics and chemistry. Science Education, 81 (4), 377-386. http:// dx.doi.org/10.1002/(SICI)1098237 X(199707)81:4<377::AID SCE1>3.0.CO;2-9

SOLBES, J. \& VILCHES, A. (2004) Papel de las relaciones entre ciencia, tecnología, sociedad y ambiente en la formación ciudadana. Enseñanza de las Ciencias, 22 (3), 337 -348.

SOLOMON, J. (1996). STS in Britain: science in a social Contex. In:Yager, R. (Org.) Science/Technology/Society as reform in science education. New York: State University.

VIEIRA, R, D.; NASCIMENTO, S. S. (2007). A argumentação no discurso de um professor e seus estudantes sobre um tópico de mecânica newtoniana. Caderno Brasileiro de Ensino de Física, 24 (2), 174-193.

VIEIRA, M.R., TENREIRO-VIEIRA, C. \& MARTINS, E. (2010). Pensamiento Crítico y literaria científica. Alambique, 65, 96-104.

VÍLCHEZ, J.E. (2009). La problemática ambiental en los medios. Propuesta de un protocolo de análisis para su uso como recurso didáctico. Enseñanza de las ciencias, 27(3), 421-432. 
ZEIDLER,D.;WALKER,K.;ACKETT,W. A. \& SIMMONS, M. (2002). Tangled up in views: Beliefs in the nature of science and responses to socios- cientific dilemmas. Science Education, 86 (3), 343 - 367. http://dx.doi. org/10.1002/sce.10025

\section{ANEXO A \\ PENSAMIENTO CRÍTICO Y CUESTIONES SOCIOCIENTIFICAS}

\section{NOMBRE:}

Edad:

La siguiente situación utilizada para caracterizar el pensamiento crítico a partir del abordaje de las cuestiones socio científicas se estructura en un formato de pregunta abierta que evalúan las competencias establecidas para este tipo de pensamiento.

Considerando la lectura de los siguientes textos, desarrolla las preguntas que aparecen a continuación:

\section{La generación de la energía eléctrica.}

Muchas de las actividades realizadas diariamente como: trabajar en el computador, ver TV, conservar las frutas y verduras, pueden depender de la energía eléctrica.

1. Observa el esquema que aparece a la derecha y describe el procedimiento que se requiere para la generación de la energía eléctrica.

2. ¿Que otros procedimientos conoces que permitan hacer girar la turbina para producir energía eléctrica?

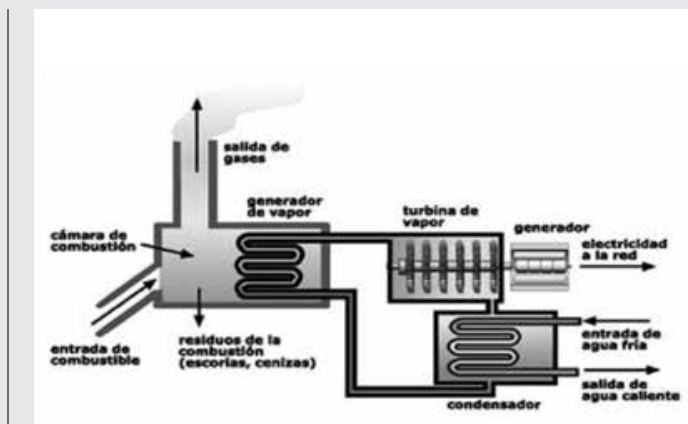




\section{Energía eléctrica en Colombia}

Considerando fenómenos de sequias asociados al fenómeno del niño hacia el año 1992 , en Colombia se incremento la utilización de la energía térmica, por lo que para el año 2011 hubo una expansión de 1,500 MW distribuida entre fuentes hídricas y térmicas. Así la producción eléctrica total en 2005 fue de 50.4 TWh. Las plantas hidroeléctricas generaron 81.2 por ciento, las plantas térmicas 18.6 por ciento y la planta eólica Jepírachi 0.1 por ciento del total.

\section{1. ¿Cómo se produce la energía eléctrica en Boyacá y quien la produce?}

Se afirma que las centrales hidroeléctricas no son contaminantes, ayudan a frenar la emisión de $\mathrm{CO} 2$ y no producen impactos ambientales

2. ¿Está de acuerdo con la afirmación anterior? ¿Cuál ha sido el impacto de las centrales hidroeléctricas en el ambiente y la sociedad? 2. ¿Y el de las térmicas?

1

2

\section{La privatización de la empresa de energía eléctrica de Boyacá EBSA}

En las últimas décadas del siglo XX, se han registrado en Colombia una tendencia hacia la privatización de las Empresas Públicas, especialmente en los sectores productivos y de servicios. El Gobierno nacional crea un nuevo marco normativo que regulaba y daba alcance al modelo neoliberal fomentado por llamado Consenso de Washington, el cual daba lineamientos de política económica a los países del tercer mundo para afrontar la nueva reestructuración del capital y pagar el servicio de la deuda. En este sentido la Constitución del 91 profundizo la descentralización política y administrativa e incentivó la rigidez de la ejecución presupuestal destinando gran parte del presupuesto nacional y 
esfuerzos fiscales a pagar la deuda externa. La inversión pública del gobierno decreció de manera sorprendente, para 1993 a 1996 la inversión en términos reales fue de $14 \%$ mientras que para los siguientes 5 años desde el $96^{\prime}$ al 2001 fue de $4.5 \%$, mostrando la imposibilidad del Estado para asumir la financiación de la inversión pública. La reestructuración del sector eléctrico vino acompañado de la "participación" de los inversionistas privados, que daba cierta credibilidad al mercado colombiano y daban acceso a créditos de la Banca Multilateral (la deuda se paga con deuda). Para 1996 se inicia el proceso de privatización de varias plantas de generación de energía, incluidas hidroeléctricas como Chivor y Betania y las termoeléctricas Termotasajero y Termocartagena. Ya en el 1997 se inicio la privatización de las electrificadoras de EPSA Empresa de Energía del Pacifico y Emgensa y la capitalización de activos de transmisión de CORELCA y de distribución de Electrocosta y Electrocaribe hoy de la Unión FENOSA ${ }^{1}$.

A partir del mes de Septiembre de 2011, varios diarios de nuestro país destacan titulares relacionados con la privatización de la empresa de Energía del departamento de Boyacá,

\section{Gobierno ratifica venta de la Empresa de Energía de Boyacá}

Bogotá, D.C., 18 de octubre de 2011 (Oficina de Prensa del Senado). El senador Juan De Jesús Córdoba (Partido Conservador), defendió la no privatización de la Empresa de Energía de Boyacá, EBSA, tras asegurar que "no se debe vender porque es una empresa rentable, económica y social".

Según el congresista, "esa acción atenta contra el patrimonio de los boyacenses, pues la empresa es un ejemplo de eficiencia en el manejo de los recursos públicos. Además se encuentra entre las cien mejores empresas del país". Durante su intervención, el legislador propuso adelantar un juicio de responsabilidad social a quienes atenten contra el patrimonio del departamento; y sostuvo que el Gobierno tiene cuatro razones para vender la empresa: las fiscales, de endeudamiento, estrategias y de rentabilidad.

$\mathrm{Al}$ respecto, el ministro de Hacienda Juan Carlos Echeverry, respondió ante el cuestionamiento del senador Córdoba y aseguró que "el valor patrimonial de la empresa se ha desvalorizado y por lo tanto es inminente buscar socios estratégicos, además la Nación no debe acumular activos que no le representen

1 Tomado de: Un balance crítico a la privatización del servicio público; en http://www.eldia.co/ index.php?option=com_content\&view=article\&id=159\&catid=27\&Itemid=11 
rentabilidad". Otra de las razones expuestas por el ministro se relaciona con la optimización de servicios, es decir "calidad a menor precio", según reiteró. Por lo tanto, "si no se venden esos activos se tiene que acudir al cobro de más impuestos", explicó Echeverry.. (Ver otros titulares) ${ }^{2}$

\section{Considerando la situación presentada, responde las siguientes pre- guntas:}

1. IIdentificas problemas en lo anteriormente descrito?. En caso afirmativo ¿Cuáles y Por qué?

2. Consideras que la situación descrita anteriormente puede ser objeto de reflexión de las ciencias

Si_ No

En caso afirmativo ¿En qué aspectos y Por qué?

3. Estás de acuerdo con la privatización de la EBSA. ¿Por qué?

4. Estás de acuerdo con la forma de producción de la energía eléctrica en el departamento de Boyacá.

${ }^{2}$ En: http://static.cdn.elespectador.com/noticias/nacional/articulo-306259-empresa-de-energiade-boyaca-sera-vendida http://revista.blog.pangea.org/?p=55243\&lang=es. http://www.congresovisible.org/agora/post/privatizar-a-ebsa-traera-como-consecuencia-el-deterioro-del-servicioanticipa-robledo/2635/ 
5. ¿Qué controversias o desacuerdos científicos relacionados con la producción y el uso de la energía eléctrica se han dado?:

6. Suponga que usted es invitado a un debate donde se discute la implementación de energía eléctrica en el país, y se requiere saber su valoración profesional respecto a la temática, ¿cuál sería tu declaración?.

7. Basándote en la declaración anterior que decisiones sería necesario asumir a nivel personal, laboral, y familiar respecto a la situación anterior.

Gracias por su Colaboración 
